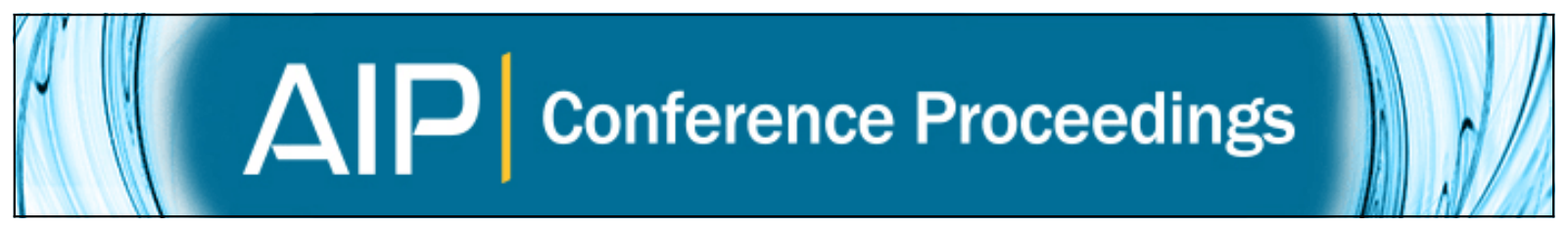

\title{
Increasing data from high rate characterization experiments using optical
} reconstruction

Clive Richard Siviour, Matthew R Arthington, Euan Wielewski, and Nik Petrinic

Citation: AIP Conference Proceedings 1426, 438 (2012); doi: 10.1063/1.3686312

View online: http://dx.doi.org/10.1063/1.3686312

View Table of Contents: http://scitation.aip.org/content/aip/proceeding/aipcp/1426?ver=pdfcov

Published by the AIP Publishing

\section{Articles you may be interested in}

Materials Characterization Using Reconstructed Thermographic Data

AIP Conf. Proc. 657, 1270 (2003); 10.1063/1.1570278

CT reconstruction from fan data using parallel backprojection

Med. Phys. 26, 2036 (1999); 10.1118/1.598709

Data Acquisition and Analysis in Extremely High Data Rate Experiments

Phys. Today 49, 50 (1996); 10.1063/1.881524

Shape reconstruction from high-frequency sonar data for robotic tasks

J. Acoust. Soc. Am. 93, 2340 (1993); 10.1121/1.406289

Optical Wave Reconstruction from Acoustically Acquired Data

J. Acoust. Soc. Am. 51, 83 (1972); 10.1121/1.1981635 


\title{
INCREASING DATA FROM HIGH RATE CHARACTERIZATION EXPERIMENTS USING OPTICAL RECONSTRUCTION
}

\author{
C. R. Siviour ${ }^{1,2}$, M. R. Arthington ${ }^{1}$, E. J. Wielewski ${ }^{1}$, and N. Petrinic ${ }^{1}$ \\ ${ }^{1}$ Department of Engineering Science, University of Oxford, Parks Road, Oxford, OX1 3PJ, UK
}

\begin{abstract}
Uniaxial characterization experiments in tension and compression are widely used to evaluate the mechanical response of materials to applied deformation over a wide range of strain rates. By taking photographs of the specimen as it deforms, it is possible to more fully characterize materials by reconstructing the specimen shape during the deformation process. This allows us to better evaluate the stress and strain in the specimen during deformation, and also provides a more rigorous validation of material models. For many materials, specimens with initially circular cross sections will evolve to a different shape; in particular, hcp metals become elliptical. We have developed a technique whereby images of a specimen during deformation (for example, from a high speed camera) from three different angles are used to reconstruct elliptical cross sections during an experiment. The technique has been applied to tensile Hopkinson bar experiments and Taylor Impact experiments on Zirconium. This paper presents an overview of the technique and the data that it can provide.
\end{abstract}

Keywords: HCP Metal, Hopkinson Bar, Taylor Impact, Optical Techniques, High Speed Photography PACS: 62.20.D- , 62.20.F-

\section{INTRODUCTON}

Over the past century, a number of techniques have been developed for characterizing the response of materials over a wide range of strain rates [1]. Up to strain rates of c.a. $10,000 \mathrm{~s}^{-1}$, the most common methods involve quasi-uniaxial loading of initially cylindrical specimens in screw driven, hydraulic or impact devices, the last of these includes the well-known split-Hopinson bar. Another technique, usually now used for validation of material models, is the Taylor Impact experiment.

Of particular interest for industrial applications is the tensile test using dog-bone specimens. A small number of researchers have performed high rate tensile tests, using the split- Hopkinson bar first implemented by Harding and co-workers [2]. Whilst tensile testing has a number of advantages over compression, such as a reduction in the effects of friction, it is not ideally suited to the traditional split-Hopkinson bar methodology, in which the forces and displacements of the specimen-bar interface are used to calculate the stress and strain in the specimen. In particular, dog-bone shaped specimens accumulate strain outside the gauge length, and calculations of true stress must account for necking in the specimen [3]. For this reason, authors have adopted high speed photography as a means to obtain more widely applicable data $[4,5]$.

This paper presents an overview of recently developed techniques for increasing the data obtained from materials characterization experiments. A new geometry reconstruction technique, which uses images of a deforming specimen taken from three angles, allows us to better measure the mechanical properties of specimens that evolve elliptical cress-sections when deformed. This extends the photographic techniques used by previous authors to materials, 
such as the hcp metals, which can have strongly anisotropic behavior. Whilst the technique can be applied to a range of experiments, this paper concentrates on tension and Taylor Impact experiments on Zirconium.

\section{EXPERIMENTAL PROCEDURE}

\section{Geometry Reconstruction}

An ellipse can be fully defined by five independent parameters, through the equation $A x^{2}+B x y+C y^{2}+D x+E y-1=0$. More usefully, these parameters can be re-expressed as the orientation $(\phi)$, major and minor radii $\left(r_{a}, r_{b}\right)$ and the location of the center $(x, y)$. The values of these parameters can be derived from tangent lines. In particular, by imaging the specimen from different angles using three cameras, six tangent lines are produced, which is one more than required, for each cross-section. The ellipse parameters can be calculated using a least-squares approach [6].

The reconstruction process, illustrated in Fig. 1 , involves taking three images of a specimen at discrete points in time during the experiments. This is done using three cameras for quasi-static experiments, or one camera (Specialised Imaging SIMX16) and two mirrors to produce three 'virtual' cameras at high rates and in Taylor impact. Extrinsic and intrinsic calibration procedures are used to define the relative position of the cameras and specimen and correct the images; following this, a digital-correlation based edge detection routine finds the specimen edges. This process works best if the images are silhouettes, although this is not a requirement. The edges are used to reconstruct the specimen geometry at all axial positions, outputting a range of data, of which the most important for this paper are $r_{a}$ and $r_{b}$ as functions of both axial location and time.

Because the specimen is moving through the camera image, it may be necessary to convert from the Eulerian frame (fixed with respect to the photographs) to a Lagrangian frame that moves with the specimen. This is especially important if it is required to find, for example, the evolution of area in an initially non-uniform specimen. This has been addressed through a technique that measures axial deformation by tracking the surface texture that is observed in the specimen edge locations [7].

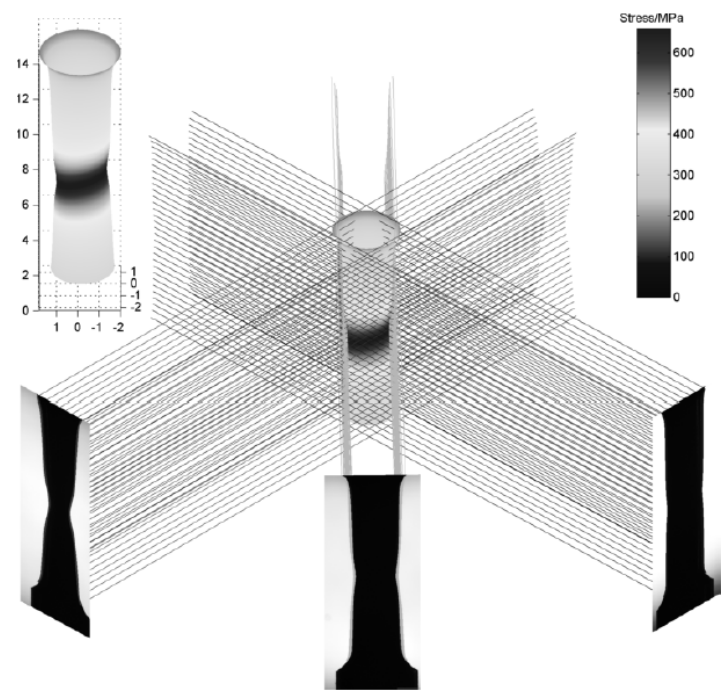

Figure 1. Overview of the reconstruction process. Three photographs are obtained from different angles. From these, the shape of the specimen is calculated. At the center and inset are the reconstructed specimen, shaded by true direct stress in MPa.

\section{Experimental Characterization}

Uniaxial tensile experiments on Zirconium were performed on specimens whose dimensions are shown in Fig. 2; these dimensions are a compromise between the requirement for stress equilibrium at high rates and the desire for a large length-diameter ratio in uniaxial testing. Quasistatic experiments were performed using a screwdriven Hounsfield testing device at a strain rate of $1.6 \times 10^{-3} \mathrm{~s}^{-1}$, while high rate experiments used the split-Hopkinson bar described in [2] at a strain rate of $4400 \mathrm{~s}^{-1}$. Nominal stress-strain curves for Zirconium are shown in Fig. 3.

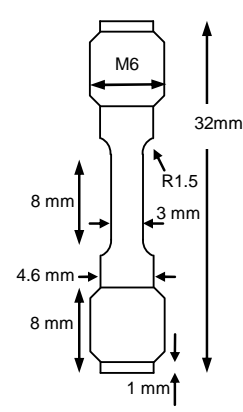

Figure 2. Tensile specimen

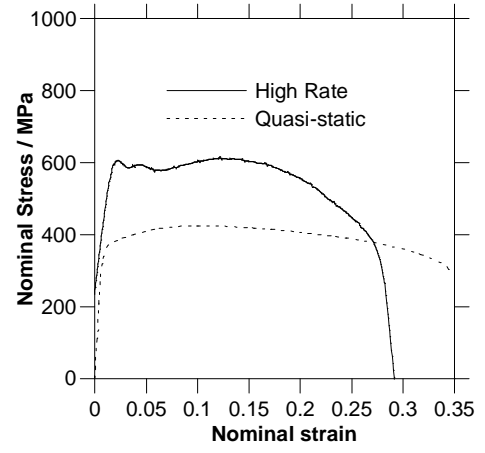

Figure 3. Nominal stressstrain curves for Zirconium 

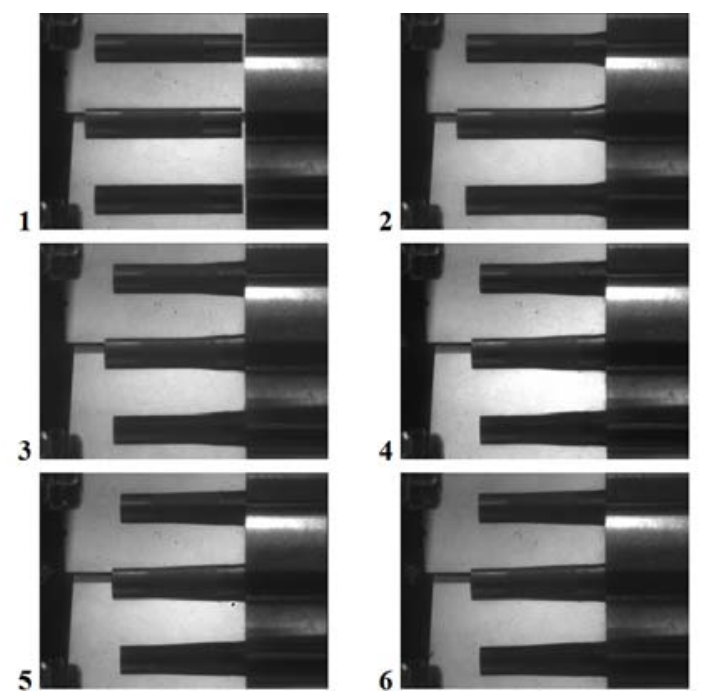

Figure 4. Sequence of pictures from Taylor impact, showing three images obtained using mirrors. Interfame and exposure times were $7 \mu \mathrm{s}$ and $1 \mu \mathrm{s}$.

Taylor Impact experiments were performed on rods of Zirconium $5 \mathrm{~mm}$ diameter and $25 \mathrm{~mm}$ long. These were impacted into a steel rod with a hardened and polished end, at speeds of about $200 \mathrm{~m} \mathrm{~s}^{-1}$. Fig. 4 shows the series of photographs obtained during one of these experiments, further information is given in [8].

\section{RESULTS AND DISCUSSION}

An example of a reconstructed tensile specimen was shown in Fig. 1. The procedure produces large quantities of data, for example Fig. 5 shows the evolution of major and minor axes of the ellipse describing the specimen shape as a function of axial position and time, whilst Fig. 6 puts the data into context by showing the shape of the most deformed part of the specimen (i.e. the center of the neck), as a function of time. A recent paper [9] validates both that the specimen does form an elliptical cross-section and the values produced by the reconstruction, through comparison with other measurement techniques.

For the Taylor impact experiment, the reconstructed geometry is shown in Fig. 7. The figure shows 'Area Strain': defined as the logarithm of the ratio of final to initial area; equal to the true longitudinal strain if the volume remains constant.

A large number of calculations may be performed on the tensile data. These include
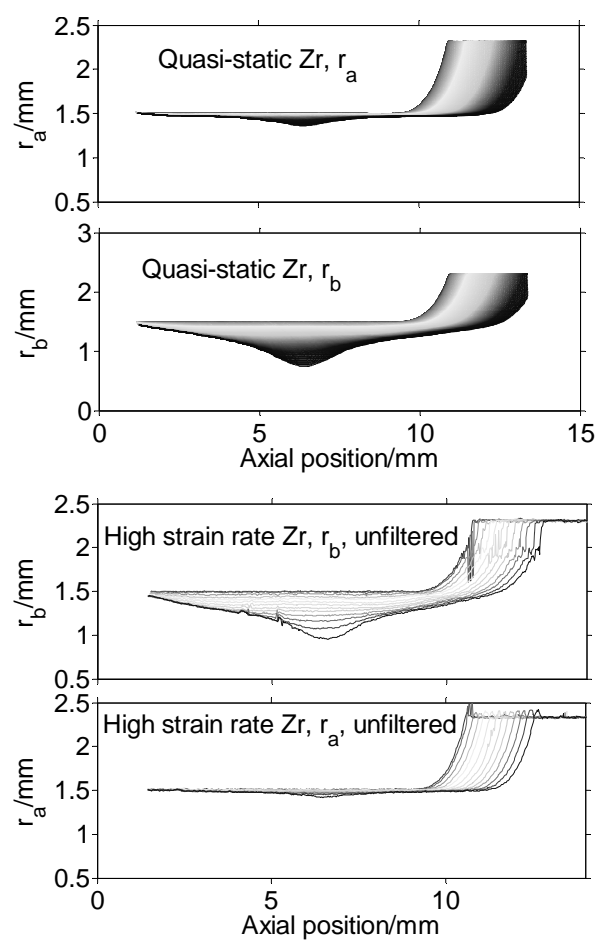

Figure 5. Major and minor radii for Zirconium as a function of axial position and time (grayscale).

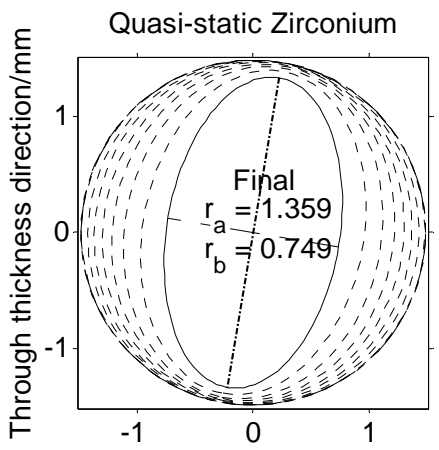

In plane direction $/ \mathrm{mm}$

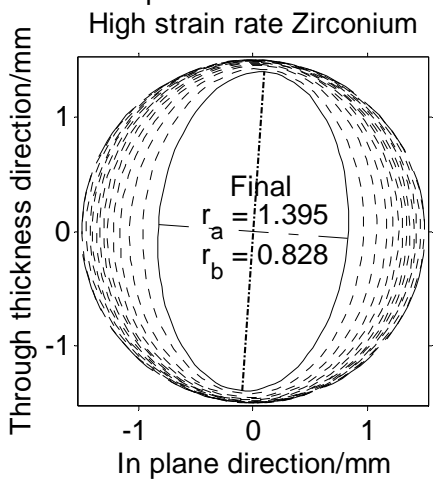

Figure 6.

Reconstructed cross-sections from different stages of the tensile experiments. 

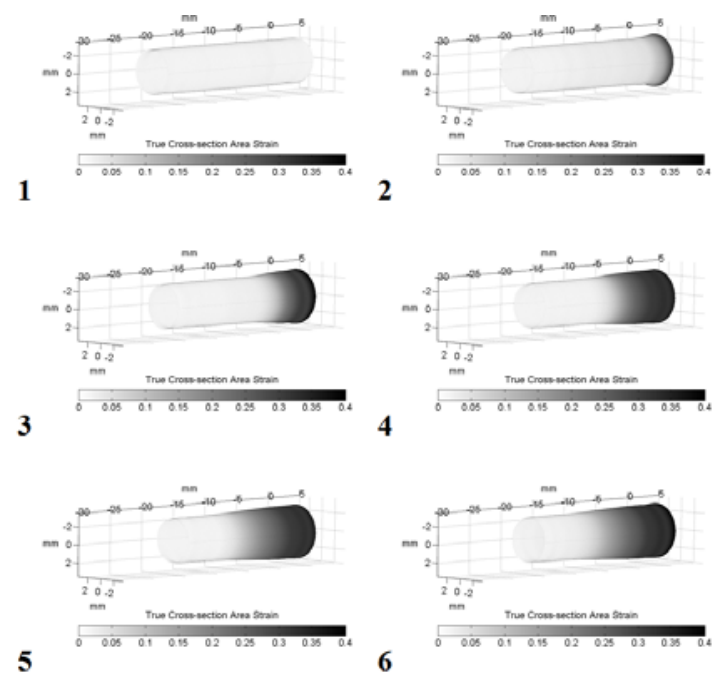

Figure 7. Reconstructed Taylor Impact specimen

accurate evaluation of area, anisotropy (e.g. the $R$ ratio [10]), true stress and radial strains; all as functions of strain rate, axial strain and position.

Furthermore, if it is assumed that the material volume remains constant, and that plane sections remain plane, axial true strain can again be calculated. These 'full-field' data can be reduced for further visualization, such as the stress-strain curves in Fig. 8, which are produced using data from the most highly deformed part of the specimen (the center of the neck) and therefore allow material properties to be calculated up to very large strains. A more thorough investigation is presented in [9].

It is important to note that in all cases, very large volumes of data are produced. Whilst the production of stress-strain curves is important for visualizing the data, and for comparisons to other measurements of material properties, the main power of the new technique will be to provide high quality characterization and validation for the development of numerical simulations over a wide range of strain rates and loading geometries.

\section{CONCLUSIONS}

A method has been presented for the reconstruction of 3D geometries of mechanical specimens that deform with elliptical crosssections. The technique has been applied to Taylor Impact and uniaxial tension experiments on Zirconium. In both cases, the reconstructed geometry and the 'area strain' as a function of

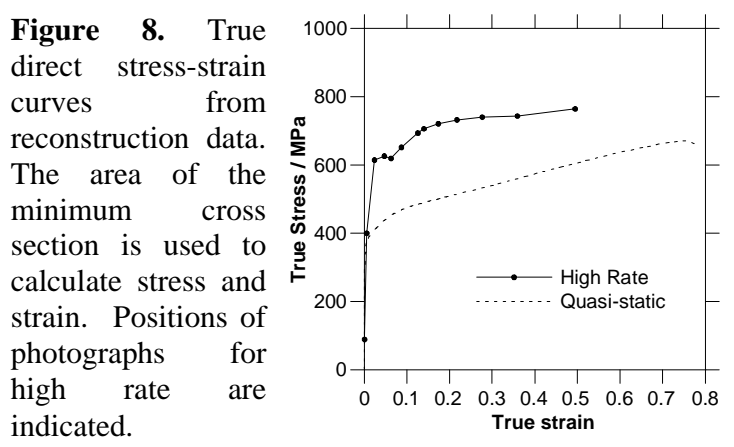

position can be calculated. In tensile tests this allows calculation of true stress-strain curves throughout the loading, giving the opportunity to generate data to very large final strains.

\section{ACKNOWLEDGEMENTS}

The authors would like to thank R Duffin, S Carter and $\mathrm{D}$ Froud for their support during these experiments. High speed photography was performed using SIM16 and SIMX16 ultra high speed cameras. The former was provided by the EPSRC Engineering Instrument pool. The authors are particularly grateful to A Walker for his advice and support. CR Siviour warmly acknowledges the support of Pembroke College towards his research.

\section{REFERENCES}

1. Field, J.E., et al, Int. J. Impact Engng, 30 pp. 725755, 2004

2. Harding, J., and Welsh, L. M., Journal of Materials Science, Vol. 18, pp. 1810-1826, 1983

3. Bridgeman, P. W., "The stress distribution at the neck of a tension specimen",Collected experimental papers, Harvard University Press, 1964

4. Noble, J.P., et al, Journal of the Mechanics and Physics of Solids, Vol. 47, pp. 1187-1206, 1999

5. Yang, H.S., et al, Int. J. Mechanical Sciences, Vol. 42, pp. 2395, 2000

6. Arthington, M. R., et al, Measurement Science and Technology, Vol. 20, 075701, 2009

7. Arthington, M.R., et al, Meas. Sci. Technol. Vol. 22, 025304, 2011

8. Wielewski, E. J., et al, Journal of Strain Analysis for Engineering Design, Vol. 45, pp. 429-437, 2010

9. Arthington, M.R. at al, Submitted to Int. J. Impact Engng

10. Kocks, U.F., et al, "Texture and Anisotropy: Preferred Orientations in Polycrystals and their Effect on Materials Properties", CUP 1998 